\title{
Estudos de Actividade Fotodinâmica de Corróis em Células Tumorais
}

\author{
JoANA F. B. BARATA*
}

\begin{abstract}
D. e forma a contribuir para o desenvolvimento de novas moléculas de tipo corrol com potencial aplicação em Terapia Fotodinâmica (PDT), foram sintetizados novos conjugados corrol-ciclodextrina. Após a determinação de alguns parâmetros que envolvem a absorção de radiação, relevantes para a acção de um fotossensibilizador, foi avaliada a potencial actividade fotodinâmica dos novos conjugados e do corrol precursor sobre uma linha celular tumoral - HeLa. As alterações celulares provocadas pelos fotossensibilizadores foram estimadas por ensaios imunológicos.
\end{abstract}

\section{INTRODUÇÃo}

A procura de novos princípios activos para as várias doenças que assolam a população mundial é um móbil para a descoberta e síntese de novos compostos. Foi esta a principal razão para o aparecimento de um novo tipo de macrociclos tetrapirrólicos aromáticos, os corróis. Este tipo de macrociclo tem suscitado elevado interesse da comunidade científica pela sua química, que é tão própria, tão especial e tão criativa.

A história dos corróis começa no estudo desenvolvido na segunda década do século XX por George Whipple. Este investigador estudou a influência da dieta alimentar, na regeneração da hemoglobina, em cães com anemia, dando particular atenção a dietas ricas em fígado. G. Minot e W. Murphy estenderam esta terapia a doentes com anemia perniciosa, obtendo excelentes resultados. A "terapia do fígado", aplicada ao tratamento da anemia perniciosa, valeu a G. Minot, G. Whipple e W. Murphy o Prémio Nobel da Fisiologia e Medicina, em 1934 [1].

No final dos anos 40, Folkers et al. [2] na procura do princípio activo desta nova metodologia, isolaram um pigmento vermelho cristalino do fígado, o qual designaram de vitamina $\mathrm{B}_{12}$.

Departamento de Química - Universidade de Aveiro Campus Santiago, 3810-193 Aveiro.

E-mail: jbarata@ua.pt
Em 1949, Pierce et al. [3] separaram duas formas cristalinas de vitamina $\mathrm{B}_{12}$, igualmente eficazes no combate da anemia perniciosa: a cianocobalamina e a hidroxicobalamina. Estudos de difracção por Raios $X$ desenvolvidos por D. Crowfoot-Hodgkins [4], elucidaram a estrutura da cianocobalamina contendo um novo tipo de macrociclo (Figura 1), valendo-Ihe o Prémio Nobel da Química em 1964 [5]. Estes estudos identificaram pela primeira vez um novo tipo de macrociclo denominado de corrina 1 (Figura 2).

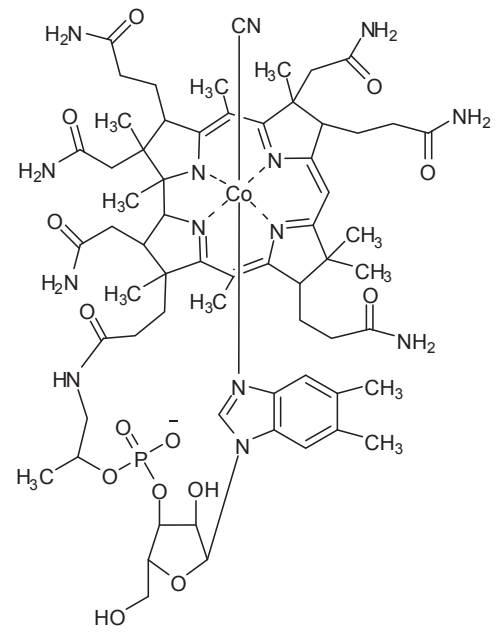

Figura 1 - Estrutura da cianocobalamina

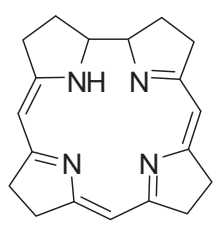

Ao examinar possíveis métodos de síntese para a preparação de corrinas, Jonhson e Price [6] descobriram uma rota sintética para preparar vários derivados metálicos de um macrociclo conjugado, que por sugestão do editor da revista (J. Chem. Soc.), denominaram de CORROL. Cinco anos mais tarde, Jonhson e Kay [7] corrigiram a estrutura proposta com onze ligações duplas e sugeriram o mesmo termo para designar a estrutura 2 (Figura 2), que contém dez ligações duplas. Os corróis são macrociclos tetrapirrólicos com uma ligação directa entre duas unidades afins do pirrol, semelhante à estrutura da corrina e mantêm a aromaticidade, tal como as porfirinas, com 18 electrões $\pi$.

Embora o macrociclo corrólico seja conhecido há mais de 40 anos, a investigação fundamental da sua química, assim como as potencialidades das suas aplicações, permaneceu quase inexplorada até à descoberta de novas rotas sintéticas desenvolvidas para meso-triarilcorróis, em 1999. Uma pesquisa rápida na literatura revela que, de todos os artigos publicados sobre corróis, desde a primeira

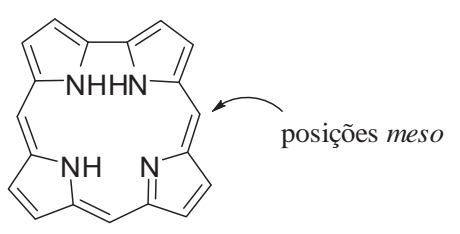

2

Figura 2 - Estruturas dos núcleos corrina $\mathbf{1}$ e corrol $\mathbf{2}$ 
publicação em 1965 , cerca de $3 / 4$ foram publicados nos últimos cinco anos.

Mas o que torna este macrociclo tão interessante? A par da capacidade que os corróis têm em estabilizar iões com elevados estados de oxidação (III, V, etc), participam em reacções de substituição [8, 9], cicloadição [10] e complexação [11], permitindo a síntese de macrociclos com propriedades úteis em várias aplicações: desde o seu uso como catalisadores, sensores e células solares, à potencial aplicação em Medicina. O facto de possuírem propriedades fotofísicas atractivas e de já serem conhecidas algumas aplicações medicinais deste tipo de macrociclo [12], possibilita o seu uso no tratamento e diagnóstico de doenças oncológicas

Das várias modalidades para o tratamento paliativo e curativo de patologias oncológicas e não oncológicas, a terapia fotodinâmica (PDT) tem revelado um grande potencial no tratamento e detecção de algumas formas de cancro, lesões pré-cancerosas e também no tratamento oftalmológico da degeneração da mácula da retina. A PDT baseia-se na combinação de três componentes não tóxicos per si: fotossensibilizador (FS), luz e oxigénio molecular (Esquema 1). Numa primeira fase é administrado o agente fotossensibilizador (de uma forma tópica ou sistémica), que após se acumular de forma selectiva em células tumorais é promovido a um estado excitado, mediante a iluminação da lesão, com luz de comprimento de onda adequado ( $\lambda \geq 600 \mathrm{~nm}$ ). Inicia-se assim uma sequência de processos fotoquímicos e fotofísicos que origina espécies citotóxicas e altamente reactivas, como os radicais e o oxigénio no estado singuleto $\left({ }^{1} \mathrm{O}_{2}\right)$, que provocam danos irreversíveis às células, conduzindo-as à morte.

As maiores vantagens da PDT sobre outros tratamentos oncológicos convencionais são: a baixa toxicidade sistémica; a habilidade de destruir tumores selectivamente, apresentando um efeito cosmético muito acentuado e o poder ser aplicada em combinação com outras modalidades terapêuticas, como a quimioterapia, cirurgia e radioterapia.
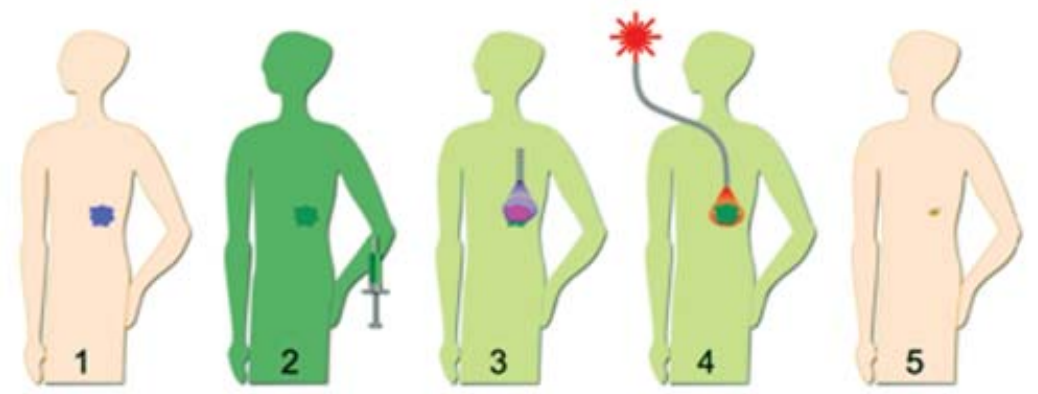

Esquema 1 - Esquema geral da Terapia Fotodinâmica (PDT)

Os fotossensibilizadores mais usados e estudados em PDT têm por base o núcleo porfirínico. A "Photofrin", uma formulação comercial constituída por dímeros e outros oligómeros porfirínicos ligados por ligações éter, éster e carbono-carbono, está aprovada para uso clínico em vários países, como os Estados Unidos da América, Canadá, Japão, Itália e Portugal.

Na procura de um fotossensibilizador ideal, entre outras propriedades [13], a anfifilia é um requisito importante, porque permite uma melhor incorporação na membrana celular [14].

De um modo geral, os corróis e as porfirinas apresentam um carácter lipofílico acentuado, exibindo baixa solubilidade em ambientes aquosos. É aceite que os macrociclos anfifílicos se acumulam, preferencialmente, nos tecidos tumorais face aos tecidos normais com um aumento considerável do efeito fotodinâmico.

\section{Síntese e Avaliação Fotodinâmica}

O objectivo do trabalho descrito foi o de contribuir para o desenvolvimento de novas moléculas de tipo corrólico com potencial aplicação em PDT. Procedeu-se ao estudo da síntese, caracterização estrutural e avaliação biológica de novas moléculas resultantes do acoplamento do corrol PFC com $\beta$-ciclodextrinas, unidades estruturais com propriedades biológicas conhecidas e relevantes, que conferem anfifilicidade [15-17].

Os novos conjugados corrol-ciclodextrina $\beta C D 1$ e $\beta C D 2$ (Figura 3) foram sintetizados por meio de uma substituição aromática nucleófila regioselectiva.
Tal como já foi referido, a PDT tira partido da acção conjugada de oxigénio, de um fotossensibilizador e de radiação de determinado comprimento de onda. Torna-se assim importante conhecer alguns parâmetros que envolvem a absorção de radiação e que são relevantes na avaliação da acção de um fotossensibilizador.

Os valores dos rendimentos quânticos de fluorescência e de oxigénio singuleto determinados estão dentro da gama dos valores normalmente usados em PDT. Verificou-se ainda, que o rendimento quântico de formação de oxigénio singuleto é superior ao da "Photofrin".

Em relação aos coeficientes de partição, os compostos $\beta C D 1$ e PFC apresentaram um carácter hidrofóbico e a sua administração nas culturas celulares foi efectuada através da sua incorporação em vesículas de lipossomas de dipalmitoilfosfatidilcolina (DPPC). O composto $\beta C D 2$ provou ser hidrófilo e foi administrado nas culturas celulares em solução aquosa [18].

Após a avaliação das propriedades dos compostos foi avaliada a potencial actividade fotodinâmica dos novos conjugados e do corrol precursor sobre uma linha celular tumoral - HeLa.

Os tratamentos fotodinâmicos consistiram em incubar as células com o fotossensibilizador, a diferentes concentrações, e depois irradiá-las com doses de luz pré-definidas, com luz de comprimento de onda determinado. A estimativa da viabilidade celular, depois dos tratamentos fotodinâmicos, foi determinada pelo teste de redução do MTT (4,5-dimetil-3-tiazolil-2,5-difeniltetrazolio) (Figura 4). 


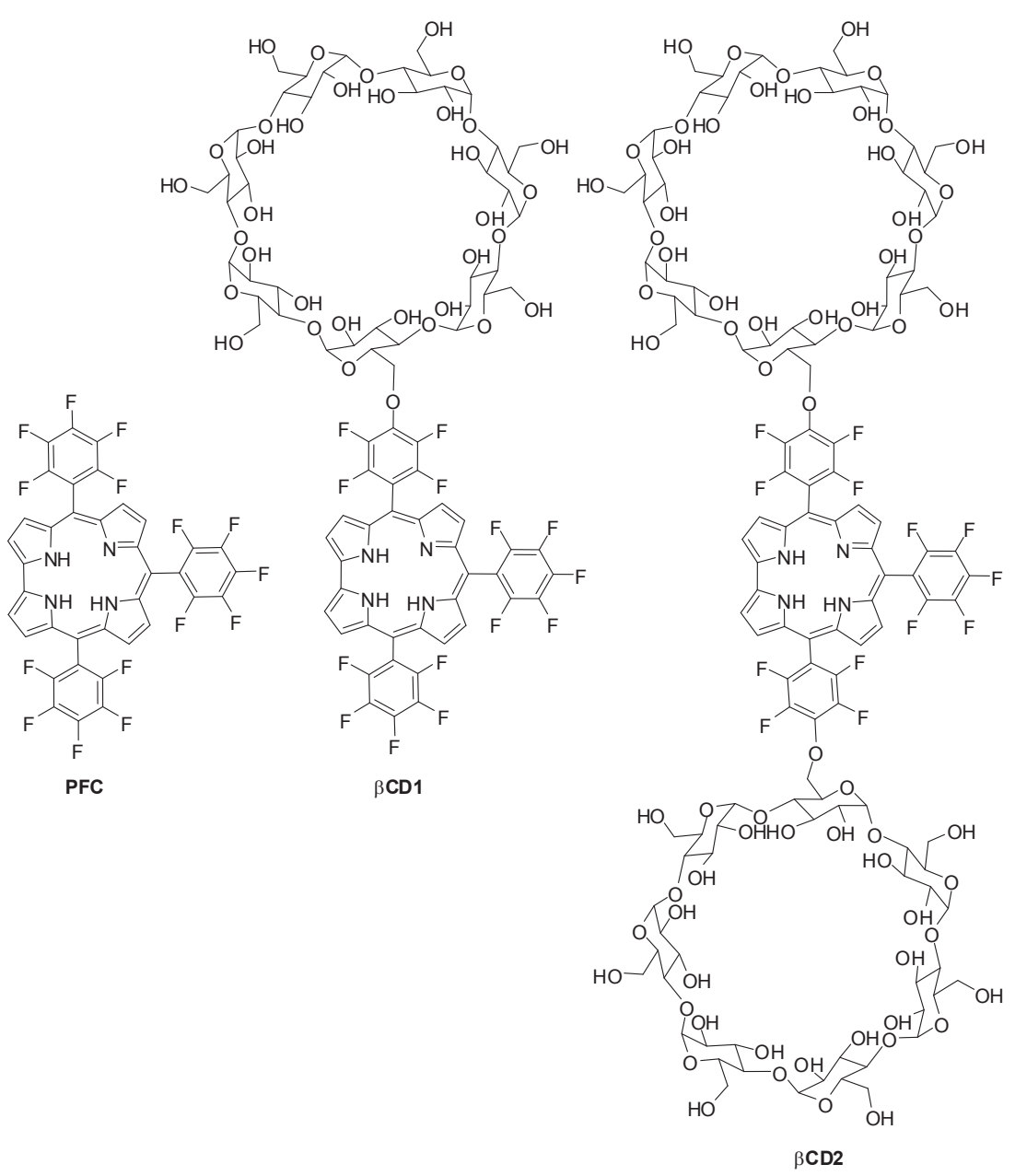

Figura 3 - Estruturas dos compostos sintetizados PFC, $\boldsymbol{\beta C D} 1$ e $\boldsymbol{\beta}$ CD2

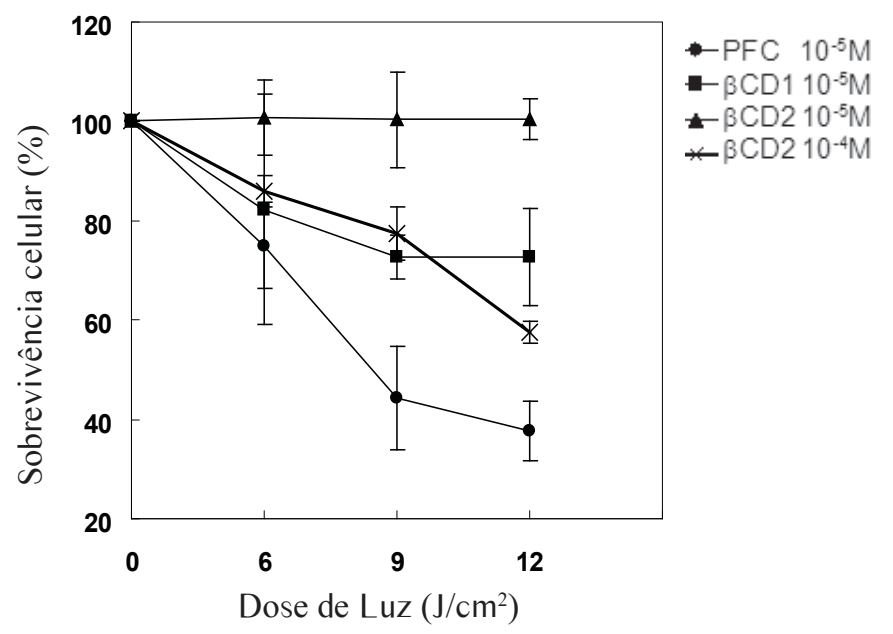

Figura 4 - Comparação dos três fotossensibilizadores

A figura 4 ilustra a percentagem de sobrevivência das células HeLa obtida pelos ensaios de redução de MTT. Pode-se observar que, tendo em conta o máximo de dose de luz utilizada (12 J/cm²), o PFC a uma concentração $10^{-5} \mathrm{M}$, foi a droga mais eficiente apresentando uma sobrevivência celular, de apenas 38\%. O $\beta$ CD1 e o $\beta C D 2$, à mesma concentração, apresentaram uma sobrevivência celular de $73 \%$ e proliferação celular.

Contudo, o composto $\beta$ CD2 a $10^{-4} \mathrm{M}$ mostrou ter um efeito moderado na viabilidade celular.
Nestes estudos, após tratamento das células HeLa com os fotossensibilizadores nas concentrações que apresentaram maior percentagem de morte celular, foram realizados ensaios imunológicos para avaliar as alterações celulares provocadas pelos fotossensibilizadores. Os ensaios foram efectuados sobre dois dos filamentos que constituem o citoesqueleto das células: os filamentos de actina e os de tubulina (microtúbulos).

Para avaliar estes efeitos, após 24 horas dos tratamentos fotodinâmicos, as preparações celulares foram preparadas, de acordo com metodologias já descritas, para cada tipo de filamento [17].

As figuras 5 e 6 apresentam as imagens de microscopia de fluorescência captadas após tratamento imunológico.

De uma forma geral, após tratamento fotodinâmico com os diferentes compostos a rede dos microtúbulos perde a sua organização. Comparando com os microtúbulos das células controlo (Figura 5a), observa-se que o composto PFC é o que provoca um maior efeito sobre as células (Figura $5 \mathrm{~d}$ ).

No caso de $\beta C D 2$, os efeitos fotodinâmicos sobre a rede de microtúbulos também são evidentes, ocorrendo a formação de células com mais que um núcleo - células poliplóides (Figura 5c).

Nas células tratadas com $\beta C D 1$ (Figura $5 \mathrm{~b}$ ), os filamentos de tubulina apresentam-se desorganizados e com perda de adesão entre células vizinhas.

De uma forma geral todos os fotossensibilizadores afectam os filamentos de actina. Comparando os filamentos de actina, após os tratamentos com os três fotossensibilizadores, com os das células controlo, estes sofrem redução, perda de organização e das propriedades de adesão ao substrato. No caso do PFC (Figura 6d), para além da perda das propriedades já referidas, observa-se ainda fluorescência relativa a actina livre, o que indica, mais uma vez, um elevado número de mortes celulares. 

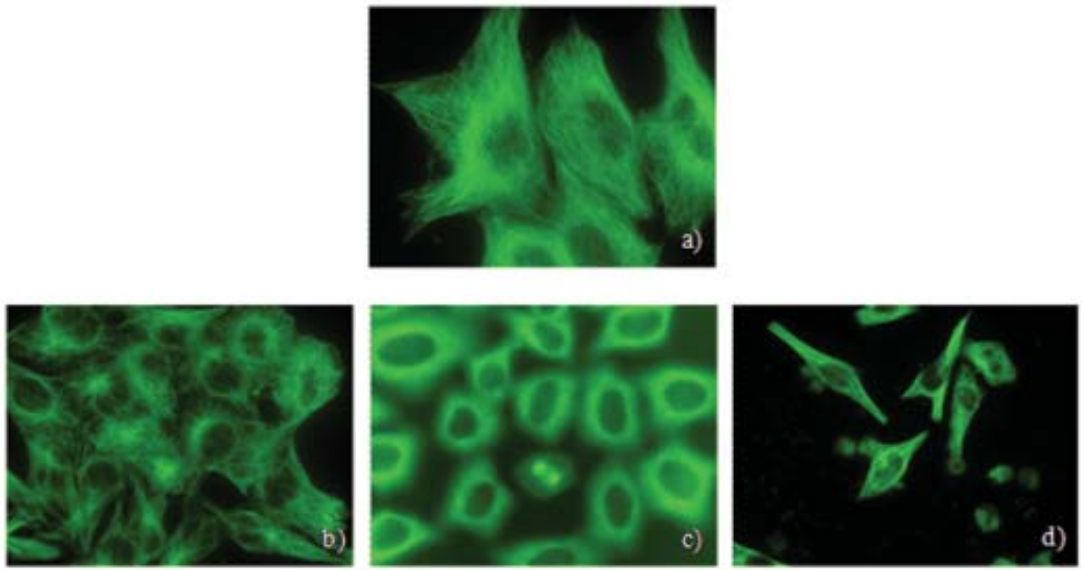

Figura 5 - Ensaios imunológicos de $\alpha$-tubulina em células HeLa: a) células controlo; b-d) após 24 h do tratamento fotodinâmico de: b) $\beta C D 1$; c) $\beta C D 2$; d) PFC

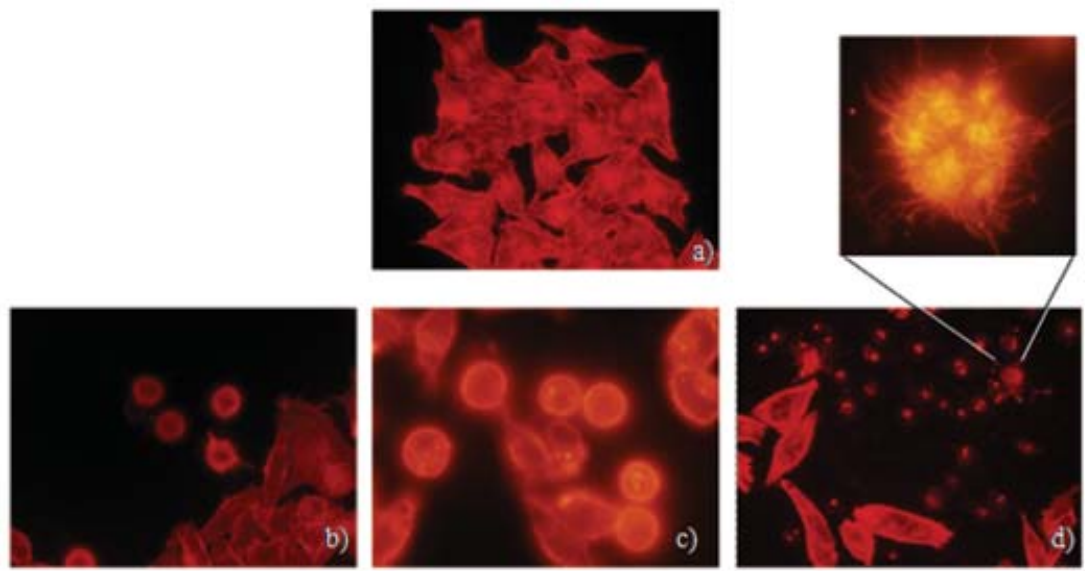

Figura 6 - Fotomodificações induzidas pelos compostos nos filamentos de actina, marcadas com faloidina: a) células controlo; b-d) após $24 \mathrm{~h}$ do tratamento fotodinâmico de b) $\beta C D 1 ; c) \beta C D 2$; d) PFC (fluorescência da actina livre está evidenciada)

\section{CONCLUSÕES}

Os resultados dos ensaios imunológicos efectuados permitem concluir que o PFC é o melhor fotossensibilizador, mas todos os compostos testados têm um efeito moderado a severo nos filamentos de tubulina e actina.

Os resultados obtidos permitir-nos-ão desenvolver novos derivados corrólicos com melhor efeito fotodinâmico.

Por esta e pelas diferentes áreas de aplicação torna-se claro que os corróis são uma classe de moléculas emergente e muito importante e que o estudo da sua química fundamental, é essencial para o desenvolvimento de novas e indispensáveis moléculas.

\section{Agradecimentos}

Desejo expressar os meus agradecimentos aos meus orientadores Prof. Dr. José A. S. Cavaleiro e Prof. Dra.
M. Graça P. M. S. Neves, à Prof. Angeles Juarranz pelo estágio realizado no laboratório de microbiologia da UAM, à Dra. Amparo Faustino em colaboração com a Prof. Beate Röder do Institut für Physik, Photobiophysik, determinação dos rendimentos quânticos de fluorescência e de oxigénio singuleto, à UA e à FCT por me ter concedido uma bolsa de doutoramento (SFRH/BD/18260/2004).

\section{REFERÊNCIAS}

[1] http://www.nobel.se/medicine/laureates/1934/press.html. 20/04/2008.

[2] Rickes, E. L.; Brink, N. G.; Koniuszy, F. R.; Wood, T. R.; Folkers, K. Science, 1948, 107, 396-397.

[3] Pierce, J. V.; Page A. C.; Stokstad, E. L. R; Jukes, T. H. J. Am. Chem. Soc., 1949, 71, 2952-2952.

[4] Hodgkin, D. C.; Kamper, J.; Maureen, Humboldt- Universität zu Berlin pela
M; Pickworth, J.;Trueblood, K. N.; White, J. G. Nature, 1956, 178, 64-66.

[5] http://www.nobel.se/chemistry/laureates/1964/press.html. 20/04/2008.

[6] Jonhson, A. W.; Price, R. J. Chem. Soc., 1960, 1649-1653.

[7] Jonhson, A. W.; Kay, I. T. J. Chem. Soc., 1965, 1620-1629.

[8] Ver por exemplo: Saltsman, I.; Mahammed, A.; Goldberg, I.; Tkachenko, E.; Botoshansky, M.; Gross, Z. J. Am. Chem. Soc. 2002, 124, 7411-7420.

[9] Barata, J. F. B.; Silva, A. M. G.; Neves, M. G. P. M. S.; Tomé, A. C.; Silva, A. M. S.; Cavaleiro, J. A. S. Tetrahedron Lett. 2006, 47, 8171-8174.

[10] a) Barata, J. F. B.; Silva, A. M. G.; Faustino, M. A. F.; Neves, M. G. P. M. S; Tomé, A. C.; Silva, A. M. S.; Cavaleiro, J. A. S. Synlett 2004, 7, 1291-1293. b) Vale, L. S. H. P.; Barata, J. F. B.; Faustino, M. A. F.; Neves, M. G. P. M. S.; Tomé, A. C.; Silva, A. M. S.; Cavaleiro, J. A. S. Tetrahedron Lett. 2007, 48, 8904-8908. c) Barata, J. F. B.; Neves, M. G. P. M. S.; Tomé, A. C.; Cavaleiro, J. A. S. J. Porphyrins Phthalocyanines, 2009, 13, 420-424. d) Vale, L. S. H. P.; Barata, J. F. B.; Santos, C. I. M.; Faustino, M. A. F.; Neves, M. G. P. M. S.; Tomé, A. C.; Silva, A. M. S.; Paz, F. A. A.; Cavaleiro, J. A. S. J. Porphyrins Phthalocyanines, 2009, 13, 358-368.

[11] Barata, J. F. B.; Barros, C. M.; Santana-Marques, M. G. O.; Neves, M. G. P. M. S.; Faustino, M. A. F.; Tomé, A. C.; Ferrer-Correia, A. J.; Cavaleiro, J. A. S. J. Mass. Spectrom. 2007, 42, 225232.

[12] Aviv-Harel I.; Gross Z., Chem. Eur. J. 2009, 15, 8382-8394.

[13] Bonnett, R. Chem. Soc. Rev. 1955 , 19-33.

[14] Bonneau, S.; Morlière, P.; Brault, D.; Biochem. Pharm. 2004, 68, 14431452.

[15] Ribeiro, A. O.; Tomé, J. P. C.; Neves, M. G. P. M. S.; Tomé, A. C.; Cavaleiro, J. A. S.; Serra, O. A.; Torres, T. Tetrahedron Lett., 2006, 47, 6129-6132.

[16] Kralova, J.; Synytsya, A.; Pouckova, P.; Koc, M.; Dvorak, M.; Kral, V. Photochem. Photobio., 2006, 82, 432-438.

[17] Szente, L.; Szejtli, J. Adv. Drug Delivery Rev. 1999, 36, 17-28.

[18] Juarranz, A.; Espada, J.; Stockert, J. C.; Villanueva, A.; Polo, S.; Domínguez, V.; Cañete, M. Photochem. Photobio. 2001, 73, 283-289. 